\title{
AN INTRODUCTION TO THE DIRECT DETECTION OF PARTICLE DARK MATTER (WIMPS)
}

\author{
L. MOSCA
}

(C.E.-SACLAY - DSM/DAPNIA/SPP)

\begin{abstract}
This is not a review talk, but a tentative introduction to the subject. After a reminder of the Dark Matter problem, the main candidates are considered, especially focusing on the WIMPs (Neutralino) detection. Among the different types of detectors, the double-detection bolometers are particularly emphasized. After some model independent considerations, the procedure to extract physical results (exclusion plots or evidence of a signal) from the measured recoil energy spectra is described.
\end{abstract}

Key words : Dark Matter, WIMPS, Neutralino, Supersymmetry (SUSY), Direct detection, Coherent (spin-independent) coupling, Spin-dependent coupling, Bolometers

\section{Introduction (The Dark Matter Problem) [1]}

Since about 70 years astrophysicists observe increasing evidence for the presence of Dark Matter (and/or Dark Energy) in the Universe.

Starting from the galactic scale (flat rotational curves in spiral galaxies) up to the largest structures in the Universe (anomalous velocity dispersion of objects in galaxy clusters) gravitational effects are observed which cannot be explained by the presence of "luminous matter" alone.

At the galactic scale, the discrepancy between the observed rotation curves and the curves calculated on the basis of known matter alone is increasing with the distance of the observed object from the center of the galaxy. In addition the ratio of Dark Matter over Luminous Matter is increasing with the scale of the considered structures.

From a theoretical point of view, two predictions are relevant to the Dark Matter/Dark Energy problem:

- the Inflation Models (mainly proposed to avoid the problem of the fantastic fine-tuning required for the initial conditions by the classical big bang model) predict that the average density of the Universe $(\rho)$ is equal to the "critical density" $\left(\rho_{c}\right)$ that is the density just corresponding to the transition between an open /closed (expanding for ever/recontracting) Universe. This prediction is usually written as: $\Omega=1$, where $\Omega=\rho / \rho_{\mathrm{c}}$.

- $\quad$ the Primordial Nucleosynthesis (taking place in between 1 sec and $1 \mathrm{~min}$ after the big bang) predicts for the fraction of baryonic Dark Matter : $\Omega_{\text {BARYONIC }}<0.1$ $(\Omega$ BARYONIC equal about 0.05 ).

\section{L.Mosca "An introduction to WIMP detection"}


So at the galaxy clusters level non-baryonic Dark Matter dominates over baryonic Dark Matter. At the galaxy level this was not evident until microlensis experiments (EROS, MACHO,...) put quite strong limits on the baryonic Dark Matter component (about 10 to $20 \%$ ), so that it is important to further investigate the non-baryonic candidates for Dark Matter as discussed in the following.

Taking into account both experimental observations and theoretical predictions (and allowing for a non-zero cosmological constant $(\Lambda)$ ), the presently most supported scenario looks like the one shown in Table 1 (see also Table 2 for the definition of the particle Dark Matter candidates anticipated here):

Table 1

\begin{tabular}{|c|c|c|c|}
\hline \multirow{5}{*}{$\Omega_{\mathrm{TOT}}=1$} & \multirow{4}{*}{$\Omega_{\text {MATTER }} \quad 0.3$} & \multirow{2}{*}{$\Omega_{\text {BARYONIC }} \quad 0.03$} & $\Omega_{\text {LUMINOUS }} \quad 0.003$ \\
\hline & & & $\Omega_{\text {DARK }} \quad 0.03$ \\
\hline & & \multirow{2}{*}{$\Omega_{\text {NON-BARYON }} \quad 0.3$} & $\Omega_{\text {нот рм }} 0.1 \rightarrow$ \\
\hline & & & $\begin{array}{c}\Omega_{\text {COLD DM } \quad 0.2 \rightarrow} \rightarrow \\
\text { WIMPs } \\
\text { (SUSY LSP = neutralino) }\end{array}$ \\
\hline & \multicolumn{3}{|c|}{$\Omega_{\text {VACUUM ENERGY }}\left(\Omega_{\Lambda}\right) \quad 0.7$} \\
\hline
\end{tabular}

\section{Dark Matter candidates}

Table 2 summarizes the main proposed candidates both baryonic and non-baryonic. Some comments are needed to understand this table.

In the baryonic sector ("Astrophysics") : White Dwarfs represent the final stage of "normal stars" (that is stars with a mass, in the main sequence, between 0.1 and 3 solar masses). Brown Dwarfs are compact objects with a mass below the ignition threshold (mass $\quad 0.08$ solar masses ), that is the minimum mass needed to start the full thermonuclear fusion cycle in the core of the object. Jupiters are hypothetical big planets with a mass of the order of the Jupiter mass. Neutron Stars and Black Holes are the final states of Supernovae, that is stars with a mass, in the main sequence, respectively larger than $\quad 3$ solar masses and 10 solar masses.

\section{L.Mosca "An introduction to WIMP detection"}


A special candidate in the baryonic sector is represented by the cold $\mathrm{H}_{2}$ gas: its presence as a halo enveloping the Spiral Galaxies, could explain simultaneously the Dark Matter problem and some other so far unexplained features of this type of galaxies (like the "gas consumption problem", etc.). The difficulty here is in the fact that molecular Hydrogen can only be detected indirectly and only few measurements are available up to now.

Table 2 Dark Matter candidates

\begin{tabular}{|c|c|c|c|}
\hline BARYONIC & \multicolumn{3}{|c|}{$\begin{array}{l}\text { Jupiters, Brown Dwarfs, White Dwarfs, } \\
\text { Neutron stars, Black Holes, } \\
\text { Cold } \mathrm{H}_{2}\end{array}$} \\
\hline \multirow{3}{*}{$\begin{array}{c}\text { NON- } \\
\text { BARYONIC }\end{array}$} & \multirow[b]{2}{*}{$\begin{array}{c}\text { THERMAL } \\
\text { RELICS }\end{array}$} & $\begin{array}{l}\text { HOT Dark } \\
\text { Matter (HDM) } \\
\text { relativistic }\end{array}$ & light $v$ ( few tens of eV) \\
\hline & & $\begin{array}{l}\text { COLD Dark } \\
\text { Matter } \\
\text { (CDM) non- } \\
\text { relativistic }\end{array}$ & $\begin{array}{l}\text { MPS } \\
\text { WIMPS (Weakly Interacting } \\
\text { Massive Particles) : } \\
\text { - massive v : } \\
\text { Dirac } \\
\text { Majorana }(\quad 20 \mathrm{GeV}) \\
\text {-SUSY particles: } \\
\text { Neutralino (Photino) }(20 \mathrm{GeV}) \\
\text { s-neutrino }\end{array}$ \\
\hline & $\begin{array}{l}\text { NON- } \\
\text { THERMAL } \\
\text { RELICS }\end{array}$ & \multicolumn{2}{|c|}{$\begin{array}{l}\text { Axions }\left(10^{-5} \mathrm{eV}\right) \\
\text { Monopoles }\left(10^{16} \mathrm{GeV}\right)\end{array}$} \\
\hline
\end{tabular}

In the non-baryonic sector ("Particle Physics"): we have first of all to distinguish particles which, in a first stage were in thermal equilibrium with radiation and then decoupled, from particles which have never been in equilibrium with radiation.

Then, in the first category, we have again to make a distinction between particles which, at the moment of their decoupling, were relativistic (Hot Dark Matter) and particles which were not relativistic (Cold Dark Matter).

From the point of view of structures formation in the Universe, the Cold Dark Matter is required to explain the formation of small structures (galaxies ) and the Hot Dark Matter is needed to explain the formation of large structures (cluster of galaxies and so on).

To the Hot Dark Matter class belong the light neutrinos $\left(v_{e}, v_{\mu}, v_{\tau}\right)$ which represent the only particle candidates already known, while their mass is still unknown: only upper limits have been determined $\left(\mathrm{m}\left(\mathrm{v}_{\mathrm{e}}\right)<7.2 \mathrm{eV}, \mathrm{m}\left(v_{\mu}\right)<170 \mathrm{keV}, \mathrm{m}\left(v_{\tau}\right)<18.2 \mathrm{MeV}\right)$. 
The Cold Dark Matter class contains essentially the Weakly Interacting Massive Particles (WIMPs). These can be massive neutrinos of either Dirac or Majorana (=selfconjugate) type or Supersymmetric (SUSY) particles (s-neutrino, neutralino).

Dirac neutrino and s-neutrino are practically excluded by Germanium and scintillator experiments, while only some model-dependent lower limits are put by LEP experiments on the masses of Majorana neutrino and neutralino.

Two other Cold Dark Matter candidates are the Cosmions (ad hoc proposed particles to solve simultaneously the Dark Matter and the Solar Neutrinos problems) and the SIMPs (Strongly Interacting Massive Particles). Cosmions are excluded by Silicium, Germanium and scintillators experiments, while SIMPs are still under investigation.

Neither Cosmions nor SIMPs candidates are supported by a fundamental theory. In particular for this reason WIMPs candidates, and especially Neutralino, the Lightest Supersymmetric Particle (LSP) of the SUSY theory, are presently preferred.

Among the non-thermal relics, two candidates are of special interest: Axions and Monopoles. Axions are particular bosons coupled to photons. They were introduced by Peccei and Quinn in the Standard Model to explain CP conservation in strong interactions. A rather narrow mass window is left by experiments around $10^{-5} \mathrm{eV}$. Monopoles are relic "topological defects" of very large mass ( $10^{16} \mathrm{GeV}$ ) predicted by Grand Unification Theories (GUT), and still under experimental investigation.

\section{How to detect wimps directly? [2]}

The WIMPS mass can range between $1 \mathrm{GeV}$ and $1 \mathrm{TeV}$ and their cross section with ordinary matter is of the "weak type" $\left(\sigma_{\text {WIMPS-N }}<10^{-38} \mathrm{~cm}^{2}\right.$ ).

Of special interest, from the point of view of Dark Matter, is the Lightest Supersymmetric Particles (LSP), the neutralino. In the simplest versions of SUSY (with R-parity conservation) the neutralino is stable, so that it is a good candidate as a relic particle in the Universe.

The neutralino is a complex object : it is in fact a linear combination of 4 particles ( 1 photino, 1 zino, and 2 Higgsinos). In addition its interaction with ordinary matter is depending on several parameters ( 7 ) which are free, to some extent, in the present formulations of the SUSY theory. These parameters controle the actual relative proportion of two basic couplings :

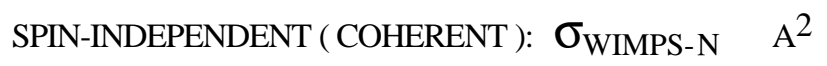

where $\mathrm{A}$ is the Atomic mass number of the target nucleus $\mathrm{N}$, and

\section{L.Mosca "An introduction to WIMP detection"}


SPIN - DEPENDENT ( AXIAL ): $\sigma_{\text {WIMPS-N }} \quad \mathrm{C} \lambda^{2} \mathrm{~J}(\mathrm{~J}+1)$

where $\mathrm{C}=\left\langle\right.$ Hadronic Matrix Element $>$ of the "odd nucleon" in the nucleus $\mathrm{N}$ and $\lambda^{2}=[\mathrm{J}$ $(\mathrm{J}+1)+\mathrm{s}(\mathrm{s}+1)-1(\mathrm{l}+1)] / 2 \mathrm{~J}(\mathrm{~J}+1)$ [Landé factor] in the single-particle Shell Model of nuclei (here $\mathrm{J}$ is the nucleus spin, while $\mathrm{s}$ and 1 are the spin and the orbital momentum of the odd nucleon).

How to detect WIMPS directly? At first sight the answer is simple: "by elastic scattering on nuclei" [3] that is by measuring the recoil energy of the scattered nucleus in the elastic reaction :

$$
\text { WIMP + N }>>\text { WIMP' + N' }
$$

But three kinds of difficulties appear immediately:

$>$ the rates of these events are small. In fact the expected events rate will depend from the type of dominant coupling and typical values are :

- one or less events/day/kg for a dominant coherent coupling (neutralino with both Zino and Higgsino(s) components, Cosmions, Dirac v, and s-neutrino), and

- less then 0.1 events/day $/ \mathrm{kg}$ for a dominant spin dependent coupling (neutralino which is mainly photino and/or zino or is pure Higgsino, and Majorana v).

$>$ the recoil energies are also small (typically from 1 to $100 \mathrm{KeV}$ )

$>$ and, last but not least, the background is comparatively very high. This is of two origins :

- the (secondary) cosmic rays which are disturbing in two ways : by directly deposing energy in the detectors and by activating the nuclei of the materials (crystals, housings, etc) composing these detectors (cosmogenic radioactivity). For this reason it is essential to install the detectors deep underground.

- the radioactive background (in addition to the component of cosmogenic origin). For that it is necessary :

to shield (with $\mathrm{Pb}, \mathrm{Cu}$, etc) the detectors from the environment to use very low activity materials (by selection and/or purification)

to maximize the intrinsic rejection power of the detectors, by Pulse Shape Discrimination (PSD) of the signals and/or by the simultaneous detection of two different physical quantities (heat and ionization or heat and scintillation).

Now the important question is: which are the possible signatures for the WIMPS direct detection? 
The WIMPS are expected to form a roughly spherical halo, essentially non rotating as a whole, enveloping our Galaxy (and much bigger than it), their speed following a MaxwellBoltzman distribution, of root mean square $300 \mathrm{Km} / \mathrm{sec}$ and their

local density in the Sun's region being $0.3 \mathrm{GeV} / \mathrm{cm}^{3}$.

The solar system instead is rotating (as all other stars) around the center of the Galaxy with a speed of $245 \mathrm{Km} / \mathrm{sec}$. As a consequence we are sweeping this "gas of WIMPS" and their speeds distribution appears to us as the convolution of a Maxwell-Boltzman distribution with the speed of the Earth. So the average speed of WIMPS, as measured in a terrestrial experiment, is : $\left\langle\mathrm{v}_{\mathrm{w}}\right\rangle \quad 360 \mathrm{Km} / \mathrm{sec}\left(\begin{array}{ll}\beta & 10^{-3}\end{array}\right)$.

Due to the revolution motion of the Earth around the Sun, the average $\left\langle v_{w}>\right.$ is not really constant but modulated between June and December, thus producing a modulation of the average WIMPS flux (and of the average nuclear recoils energy) of the order of 5 to $10 \%$.

This annual modulation potentially represents an important signature of WIMPS showing in this way their astrophysical origin.

An additional signature can be obtained by considering that the events rate is depending on the type of nuclei used as targets, in a way predicted by the theory.

\section{Different types of detector}

We consider first the classical semiconductor detectors (Germanium and Silicium), at liquid $\mathrm{N}_{2}$ temperature, then the crystal and liquid scintillators at room temperature and then the Bolometers operating at very low temperature (few millikelvins) which represent the most ambitious approach to the WIMPs detection.

Other types of detector at the R\&D stage, such as superconducting grains, superheated liquids (freon bubbles), ancient mica and Track Projection Chambers (TPC), are not described here.

\section{1. "Classical" Semiconductor Ge (Si) detectors}

The basic working principle of these detectors is that a crossing ionizing particle or radiation creates $\left(\mathrm{e}^{-}\right.$, hole) pairs in the crystal, then collected by an electric field $(1 \mathrm{kV} / \mathrm{cm})$ and so producing a signal which is a measure of the deposited energy. Since several years Germanium crystals are used as powerful $\gamma$ and $\beta$ detectors, mainly because of their impressive resolution ( $1 \mathrm{KeV}$ at $1 \mathrm{MeV}$ ) due to the small value of the energy needed to create an (e-, hole) pair ( $3 \mathrm{eV})$. One remarkable application is in the $2 \beta$ experiments $(\mathrm{MeV}$ energy range), because in this case $\mathrm{Ge}$ is at once the source and the detector $\left({ }^{76} \mathrm{Ge}\right.$ isotope). 
Then these detectors have been optimized for Dark Matter (WIMPs) search (energy range of a few $\mathrm{KeV}$ ). The remarkable performances of the Heidelberg-Moscow detector have to be stressed, although its energy threshold is higher then in some of the older experiments. The Heavy Dirac neutrino candidate is largely excluded by these results.

Limitations of these detectors are the microphony problem at low energies, the electronic noise and, last not least, the absence of background rejection power.

\subsection{Scintillation detectors}

The basic set-up in this case is a crystal (or liquid) scintillator emitting light when crossed by an ionizing particle or radiation. This light is collected by one or more photomultipliers, coupled to the scintillator directly or through some light guides. Typical values of the sensitivity are 5 to 10 photoelectrons per $\mathrm{KeV}$.

In spite of their poorer resolution compared to Ge detectors, scintillators present some other interesting features. Scintillation detection is a well known and simple technique (usually no cooling needed), the ratio cost/mass is reasonably low, and a wide choice of target nuclei is currently available.

In particular some of the scintillators (e.g. $\mathrm{NaI}(\mathrm{Tl})$ ) have a good light output and a possible (also if difficult at low energy) Pulse Shape Discrimination (PSD), as well as non zero spin target nuclei $\left({ }^{23} \mathrm{Na},{ }^{127} \mathrm{I},{ }^{19} \mathrm{~F},{ }^{129} \mathrm{Xe}, \ldots . ..\right)$. Other specific reasons of interest are the very high predicted axial coupling for the ${ }^{19} \mathrm{~F}$ nucleus and the high estimated Quenching Factor of liquid Xenon. (The Quenching Factor (QF) for nuclear recoils is here the scintillation efficiency relative to that of electrons of the same kinetic energy).

\subsection{Bolometers}

From the ancient Greek : $\beta \mathrm{o} \lambda \eta^{\prime}$ (= ray, lit.: something thrown), this term is presently used to designate very sensitive thermometers (including, by extension, the associated heat absorber) operating at very low temperature ( $10 \mathrm{mK})$. The reason for going down to so low temperatures follows from the basic relation:

$$
\Delta \mathrm{T}=\Delta \mathrm{E} / \mathrm{C}(\mathrm{T}) \quad \text { where }: \mathrm{C}(\mathrm{T}) \quad \mathrm{T}^{3} \quad \begin{aligned}
& \text { for pure dielectric crystals and } \\
& \text { superconducting metals }
\end{aligned}
$$

( $\mathrm{T}$ is the temperature in absolute scale, $\Delta \mathrm{E}$ the deposed energy, $\Delta \mathrm{T}$ the consequent temperature increase and $\mathrm{C}(\mathrm{T})$ the specific heat of the crystal). Typical crystals are Saffire $\left(\mathrm{Al}_{2} \mathrm{O}_{3}\right)$, Germanium, $\mathrm{LiF}, \mathrm{TeO}_{2}$, etc.

\section{L.Mosca "An introduction to WIMP detection"}


Since 1984 (Fiorini, Coron, ...) the problem of detecting the heat deposited by individual elementary particles is basically solved (remind that $1 \mathrm{KeV}$ of deposited energy $1.6 \times 10^{-16}$ Joule!).

Why using bolometers to detect WIMPs ?

As already stressed in section III, the main challenge is the suppression and/or rejection of the radioactive background. To do that the maximum information is highly desirable, while in usual detectors the heat, which represents a large fraction ( $2 / 3$ in Silicium) of the deposited energy, is generally lost. In addition the quanta of thermal energy (phonons) are very tiny $\left(10^{-4}-10^{-5} \mathrm{eV}\right)$ so a potentially very low threshold and very good resolution are expected.

Moreover the possibility of measuring simultaneously another form of deposited energy (ionization or scintillation) makes these detectors especially adapted to background rejection.

Started by the CDMS (Berkeley-Stanford) group, the ionization-heat bolometer technique is also operational in the EDELWEISS (French) experiment based in the Laboratoire Souterrain de Modane (LSM). The attached figure shows the impressive separation power, between nuclear and electron recoil events, of a massive $(320 \mathrm{~g}) \mathrm{Ge}$ ionization-heat bolometer. This is exposed to a source of both neutrons (producing nuclear recoils, so simulating WIMPs interactions) and $\gamma$ 's (producing electrons recoils, as it is the case with residual radioactivity when the source is removed). 


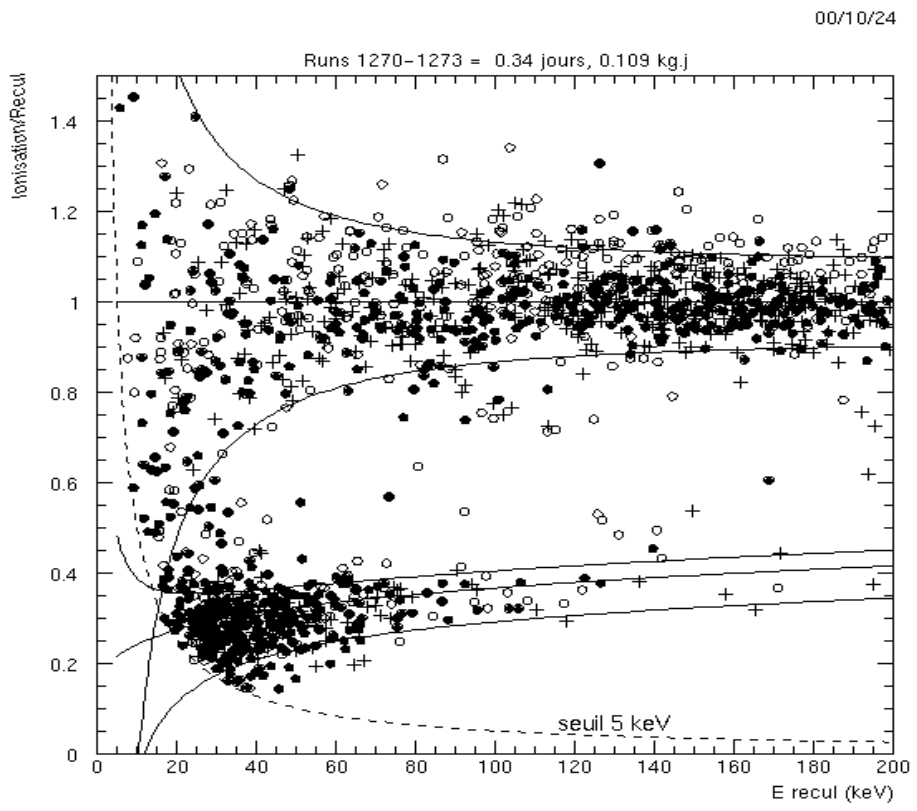

Fig. 1 Neutron and $\gamma$ calibration of a $320 \mathrm{~g}$ Germanium ionization-heat bolometer of the EDELWEISS Collaboration, exposed to $a^{252} \mathrm{Cf}$ radioactive source. The neutron events are centered around the value $Q=0.3$ (and revents around the value 1) of the Quenching factor (here defined as the ratio of the ionization energy on the recoil energy). In other words, the ionization efficiency of a nuclear recoil is about 1/3 of the ionization efficiency of an electron recoil of the same kinetic energy.

On the other side, the CRESST (Munich-Oxford) group is successfully developing a method of measuring both heat and scintillation in $\mathrm{CaWO}_{4}$ crystals.

\section{DATA ANALYSIS ( from energy spectra to exclusion plots or signal detection)}

Whatever the detector considered, the experimental results consist of measured spectra of the energy deposited in the absorber of the detector. The purpose of the analysis is to extract from these spectra a possible evidence for a WIMP signal or, at least, a so called 'exclusion plot', that is the region of WIMP cross section and mass that the experiment allows to exclude at a given confidence level.

What we already know in this process belongs to two sectors :

\section{L.Mosca "An introduction to WIMP detection"}


- astrophysics tells us essentially the global mass of Dark Matter (baryonic and nonbaryonic) present in a given region $(<R)$ of our galaxy, the local density $\rho$ as a function of the distance $\mathrm{R}$ from the center of the galaxy (equal to about $0.3 \mathrm{GeV} / \mathrm{cm} 3$ in the region of the solar system), the average speed of WIMPs ( $360 \mathrm{Km} / \mathrm{sec}$ ) and their escape velocity from the galaxy (about $700 \mathrm{~km} / \mathrm{sec}$ in the region of the solar system)

- nuclear and particle physics tells us the shape of the nuclear recoil energy $\left(\mathrm{E}_{\mathrm{R}}\right)$ spectrum as a function of the WIMP mass $\left(\mathrm{M}_{\mathrm{W}}\right)$, while its normalization depends on the WIMP-nucleon cross section, the local WIMPs number density and theWIMPs average speed:

$$
\begin{aligned}
& \mathrm{dN} / \mathrm{dE}_{\mathrm{R}} \quad\left\langle\mathrm{v}_{\mathrm{W}}>(\rho / \mathrm{M})_{\mathrm{W}}\left(\sigma_{\mathrm{W}-\mathrm{n}}\right) \exp \left(-\mathrm{E}_{\mathrm{R}} /\left\langle\mathrm{E}_{\mathrm{R}}>\right)\right.\right. \\
& \text { where : }\left\langle\mathrm{E}_{\mathrm{R}}>\quad \mathrm{M}_{\mathrm{n}}\left(\mathrm{M}_{\mathrm{W}} /\left(\mathrm{M}_{\mathrm{n}}+\mathrm{M}_{\mathrm{W}}\right)\right)^{2}\right.
\end{aligned}
$$

What is unknown and so needs to be investigated are the WIMP's mass and the cross section of its interaction with ordinary matter.

First of all, some simple remarks can be made independently of any specific model:

$\alpha)$ the slope of the recoil energy distribution $\left(1 /\left\langle\mathrm{E}_{\mathrm{R}}\right\rangle\right)$ decreases when the WIMP mass $\left(\mathrm{M}_{\mathrm{W}}\right.$ ) increases

$\beta$ ) since the total amount (mass) of the WIMPs dark matter is fixed, their number density is inversely proportional to the WIMP mass $\left(\mathrm{M}_{\mathrm{W}}\right)$

$\gamma$ ) for a fixed (measured ) events rate, the WIMP cross section is proportional to the WIMP mass : this explains the well known general behaviour of the exclusion plots.

Now to allow a comparison between experiments using different detectors, where the target nuclei are in general different (and between experimental results and theoretical predictions), the usual method is to extract from the energy spectra of each experiment cross section values (or limits) for WIMP-proton interaction, whatever the target nucleus of the specific experiment.

For that we have to distinguish the two possible types of coupling : coherent (spin independent) and spin dependent. By using respectively formula (1) and formula (2) (their complete expressions are given, for example, in ref.[4]) we can derive the following transformation relations (with quite obvious notations for the WIMP-proton (or WIMPNucleus) reduced masses and for coherent $(\mathrm{CH})$ or spin dependent (SD) form factors (FF)) : 


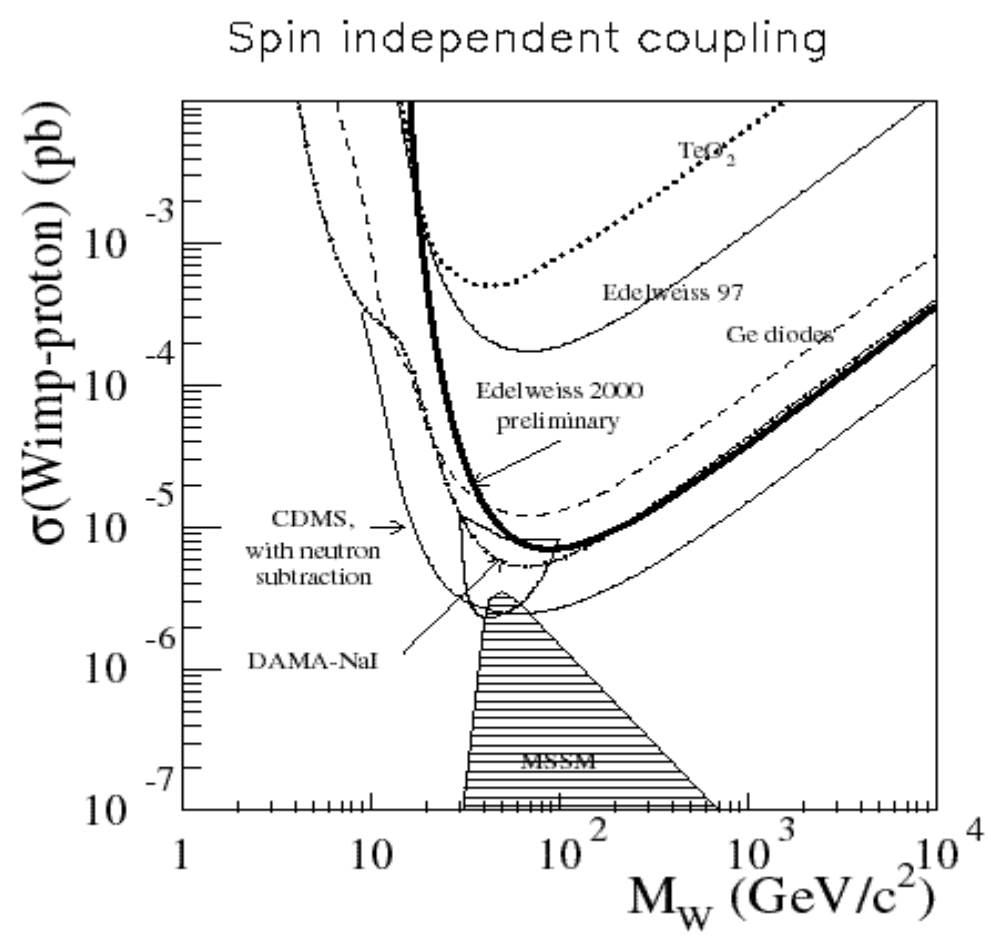

Fig. 2 Exclusion plots for the case of spin independent coupling, as obtained in some of the running experiments. (The closed curve indicates the region where an evidence for a signal is claimed by the NaI scintillator DAMA experiment).The upper region of the SUSY(MSSM) theory predictions is represented by the hatched area.

$\sigma_{\mathrm{W}-\mathrm{p}}($ spin indep. $)=\left(\mathrm{m}_{\text {red }}^{2}(\mathrm{WIMP}-\mathrm{p}) /\left(\mathrm{m}_{\text {red }}^{2}(\mathrm{WIMP}-\mathrm{N}) \mathrm{A}^{2} \mathrm{FF}_{\mathrm{CH}}\right)\right) \sigma_{\mathrm{W}-\mathrm{N}}($ spin indep. $)$

(6)

$\sigma_{\mathrm{W}-\mathrm{p}}(\operatorname{spin}$ dep. $)=\left(\mathrm{m}_{\text {red }}^{2}(\mathrm{WIMP}-\mathrm{p})\left(\lambda^{2} \mathrm{~J}(\mathrm{~J}+1)\right)_{\mathrm{p}} /\left(\mathrm{m}_{\text {red }}^{2}(\mathrm{WIMP}-\mathrm{N})\left(\lambda^{2} \mathrm{~J}(\mathrm{~J}+1)\right)_{\mathrm{N}} \mathrm{FF}_{\mathrm{SD}}\right)\right)$ $\sigma_{\mathrm{W}-\mathrm{N}}($ spin dep.)

Then we have to compare the expression (4), coupled with the relation (6) (or (7)), with the experimental spectrum for each value (interval) of the WIMP mass and deduce the corresponding value for the lower limit of the WIMP cross section. In such a way we obtain two curves (one for each type of coupling), usually called 'exclusion plots'.

\section{L.Mosca "An introduction to WIMP detection"}


An example of results of this exercise is given in fig. 2 by the exclusion plots for the case of spin independent coupling, obtained in some of the running experiments.

To investigate a possible evidence for a WIMP'signal, first it is necessary to minimize the intrinsic radioactive background (due both to photons and to neutrons) and/or reject it as much as possible. Then, if some candidates subsist, then the two signatures discussed in section 3 (annual modulation and multiplicity of target nuclei) allow to check the reliability of these candidates.

\section{Conclusion and outlook}

The WIMPs (Neutralino) detection has a three-fold fundamental purpose : 1) contribute to solve the Dark Matter problem, 2) supply a mechanism for the galaxies formation 3) support the SUSY theoretical approach.

The search in this field is now at a 'turn over' because it starts to probe the SUSY parameters predicted region.

To go deeper inside this region, new efforts on the detectors development are needed in order to:

- further decrease their intrinsic background

- further improve their rejection power against both residual radioactivity, producing electron recoils, and residual neutrons, producing nuclear recoils in the detector (as WIMPs are supposed to do!)

- increase their mass to the ton scale

and all that deep underground.

\section{References}

1. For a more general approach of the Dark Matter problem, see for ex. the book : "Basics of Modern Cosmology" by A.D. Dolgov, M.V. Sazhin and Ya.B. Zeldovich, Ed. Frontières (1990)

2. For a general presentation of the Dark Matter detection method, see for ex. P.F. Smith and J.D. Lewin, Phys. Rep. 187 (1990)

3. M.W. Goodman and E. Witten, Phys. Rev. D31 (1985) 3059

4. A. Bottino et al., Phys. Lett. B295 (1992) 330 